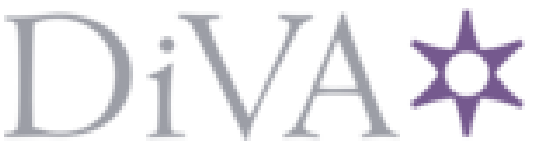

http://www.diva-portal.org

\title{
Postprint
}

This is the accepted version of a paper presented at IEEE international conference on robotics and automation, ICRA 2OO1, Seoul, South Korea, 21-26 May, 2001.

Citation for the original published paper:

Lilienthal, A J., Wandel, M., Weimar, U., Zell, A. (2001)

Sensing odour sources in indoor environments without a constant airflow by a mobile robot

In: Proceedings: IEEE international conference on robotics and automation (pp. 4005-4010). IEEE

https://doi.org/10.1109/ROBOT.2001.933243

N.B. When citing this work, cite the original published paper.

Permanent link to this version:

http://urn.kb.se/resolve?urn=urn:nbn:se:oru:diva-4016 


\section{Sensing Odour Sources in Indoor Environments Without a Constant Airflow by a Mobile Robot}

\author{
Achim Lilienthal, Andreas Zell \\ University of Tübingen, WSI-RA, \\ Sand 1 , \\ D-72076 Tübingen \\ \{lilien,zell $\} @$ informatik.uni-tuebingen.de
}

\author{
Michael Wandel, Udo Weimar \\ University of Tübingen, IPC, \\ Auf der Morgenstelle 8, \\ D-72076 Tübingen \\ \{mw,upw\}@ipc.uni-tuebingen.de
}

\begin{abstract}
A mobile robot with gas sensors commonly denoted as 'electronic nose' enables some interesting applications including the development of an 'electronic watchman' that is able to detect and localize odour sources. This paper describes the assembly of a mobile odour sensing system and presents investigations on its practical operation in an indoor environment without a constant airflow ${ }^{1}$. Lacking a constant airflow leads to a problem which cannot be neglected in real world applications. In this case the response of the used metal oxide gas sensors is dominated by air turbulence rather than concentration differences. In the following article we show that this kind of problem could be overcome by driving the robot with a constant speed, thus adding an extra constant airflow relative to the gas sensors location. If the robot's speed is not too low the described system proved to be well suited to detect even weak odour sources.

Because driving with constant speed is an indispensable condition to perform the basic tasks of a mobile odour sensing system, a new localization strategy is proposed, which takes this into account.
\end{abstract}

\section{Introduction}

The ability of an autonomous mobile robot to interact with a dynamic environment is based upon its ability to sense relevant qualities of the surrounding world. Different types of sensors are available to perform this task. Their design is often inspired by biological principles: For instance, sensors based on three of the five human senses are widely used

\footnotetext{
${ }^{1}$ This work is part of the project 'Senses for Mobile Robots' which is supported within a pinpoint program by the state of Baden-Württemberg.
}

enabling robots to 'see' (camera systems), 'hear' (microphones) and 'feel' (tactile sensors). Until now less attention was paid to the remaining senses. Merely basic investigations were carried out in the field of 'electronic tongues' [1] whereas gas sensors denoted as 'electronic noses' [2] are well established under laboratory conditions $[3,4,5]$ but have been rarely used in robotics $[6,7,8]$ so far. This might be caused by the difficulties that arise when these sensors are used in a real world scenario without the certainty of accurately defined conditions. In contrast to laboratory experiments, where usually a special odour delivery system transfers the odour from the source material to the sensor chamber, many parameters like temperature, humidity and strength of flow can vary within a comparably broad range. It is desirable to solve these problems, because there are a lot of possible applications for robots equipped with a sense of 'smell': First one can imagine an 'electronic watchman', who is able to detect and localize odours indicating a fire or a chemical hazard. Besides handling existing odours, such robots could also use self-produced odourous markers to aid navigation $[9,10]$ or to communicate with other robots [11].

However, because of the above mentioned problems most publications concerning the use of electronic noses on autonomous mobile robots are confined to the task of detecting a known gas rather than recognizing an unknown gas. Furthermore the published experiments we know of were undertaken in environments with a strong constant airflow (about $0.3 \mathrm{~m} / \mathrm{s})[7,6]$ or have been restricted to small distances between the odour source and the detector [8]. In this way, a major problem that restricts the benefit of the gas sensors which were used in the real world experiments described below, is avoided: Air turbulences, even small indiscernible air movements in unventilated apartments, make it difficult 


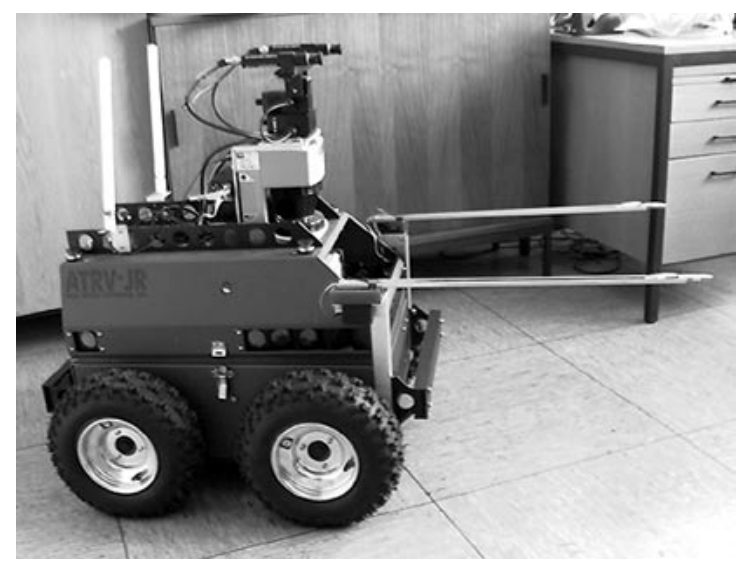

Figure 1: The autonomous mobile robot ARTHUR equipped with an electronic nose. Notice the tubeshaped sensor sticks, which were mounted at the end of the outstanding bars on the front side of the robot.

to detect spatial and temporal differences in odour molecule concentration [8, 12].

Thus the goal of this work was mainly to demonstrate that a robot equipped with an electronic nose is capable of detecting an odour source in an indoor environment without the presence of a strong constant airflow. This should be achieved using rather small odour sources which yield gas concentrations comparable to small puddles of leaking liquid chemicals. Lacking a constant airflow, it is impossible to localize the odour source by means of the two step strategy supposed by Nakamoto et al. [6] and Russell et al. [7] which involves an upwind-search after plume acquisition. Therefore, a different localization strategy is proposed in this paper.

\section{The Odour Sensing Robot}

The commercial odour sensing system VOCmeter Vario $^{2}$ was mounted on ARTHUR, a mobile robot based on the model ATRV-Jr from RWII ${ }^{3}$ (see fig.1). Because of its small size $\left(19 \times 12 \times 6 \mathrm{~cm}^{3}\right)$ the basic unit could be placed inside the robot's body. Therefore this device, which is depicted in fig. 2 can not be seen in fig.1. It gathers measured values of up to 8 single sensor sticks at a rate of $1 \mathrm{~Hz}$ and transfers recorded values to the host computer via an RS232 interface. The sensor sticks, which have been connected with the VOCmeter Vario over a thin coax ca-

\footnotetext{
${ }^{2}$ www.motech.de/pdf/Vario.pdf (MoTech, Reutlingen, Germany)

${ }^{3}$ www.rwii.com (RWII, USA)
}

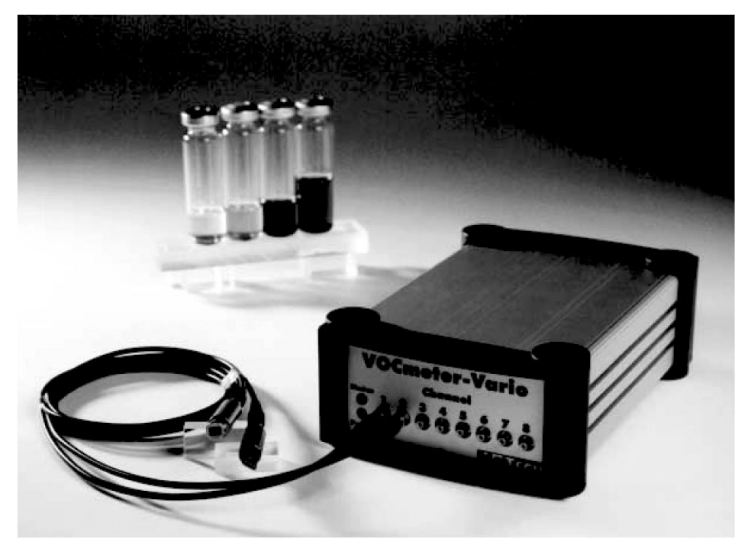

Figure 2: The commercial odour sensing system VOCmeter Vario ${ }^{2}$. The basic unit of the used system can be seen, which is able to operate up to eight sensor sticks over thin coax cables like those shown in this picture. Notice that this device can not be seen in fig. 1 because it is placed inside the robot's body.

ble (RG158) are tubes $50 \mathrm{~mm}$ long with a diameter of $10 \mathrm{~mm}$ made of high-grade steel. They not only contain the actual sensor unit but also the required transducing electronics. This system has been chosen because of its compact design, low power consumption and the facility to easily change particular gas sensors. It provides a flexible setup which, for concerns of the presented investigations, has been optimized to detect a beforehand determined substance.

For experimental simplicity ethanol and acetone have been chosen for the odour source. Experiments were undertaken using tin oxide gas sensors (Figaro ${ }^{4}$ TGS2620) because of their high sensitivity to those analytes. This type of chemical sensor show a decreasing resistivity due to an increasing amount of combustible volatile chemicals in the air. This behaviour is caused by an increasing rate of oxidation reactions taking place at the heated surface of the chemical sensitive layer. It is important to keep in mind that the functionality of the used metal oxide (MOX) sensors heavily depends on the surface temperature and that combustion of some analyte molecules (and therefore consumption of analyte material) is necessary to operate this type of sensors.

\footnotetext{
${ }^{4}$ www.figarosensor.com (Figaro, Japan)
} 


\section{The Experimental Setup}

In search of weakly ventilated locations we decided to perform two series of measurements at two different locations. First, a vacant apartment in an old building was used. Including a $20 \mathrm{~m}$ long and $2.5 \mathrm{~m}$ wide corridor, not ventilated at all and without any people passing by, this site proved to be well suited for our concerns. Because some vacant apartment odours could be sensed with the human olfactory system we decided to repeat the experiments at a different location to examine possible influences of these uncontrollable odours. The second series of measurements was performed within a similar corridor in a university building. Though the dimensions of the corridors are comparable $(30 \mathrm{~m}$ long and $2.2 m$ wide) some minor differences exist between the two used locations: The corridor at the university is weakly ventilated, one end is completed by a frequently used door and it was not possible to avoid people walking by (even at late evening hours).

In every run the robot was ordered to drive up and down while keeping track the corridors center. Thus the described scenery framed a one dimensional axis on which the odour source was placed as well. Each measuring run started with some 'patrol drives' without an extra odour source to record the odorous background at that time. In fact these reference measurements couldn't be started until the MOX sensors reached their thermal equilibrium, which requires a warm up time of about an hour. Then a jar containing liquid ethanol or acetone was opened. In order to simulate odour sources with various intensities three different jars were used with an opening area of 20,60 and $130 \mathrm{~cm}^{2}$

First the jars were placed at the end of the corridor. This means that due to the implemented obstacle avoidance the minimum distance between the MOX sensors and the odour source equaled approximately $50 \mathrm{~cm}$.

Using the smallest jar established another experiment: The robot is able to roll over this flat container, thus avoiding the need to stop in front of the odour source. Therefore, further series of experiments could realized with an odour source placed in the middle of the corridor and not at the end of it.

In order to optimize the performance of the system, three sensor positions were tested: The sensor sticks could be fixed directly to the robot on the outer end of its rear or front bumper. Moreover, the robot was equipped with a pair of 'antennae'. These bars made of aluminium were $60 \mathrm{~cm}$ long. Controlled by common servo motors, they could be rotated about a vertical axis near the robot's

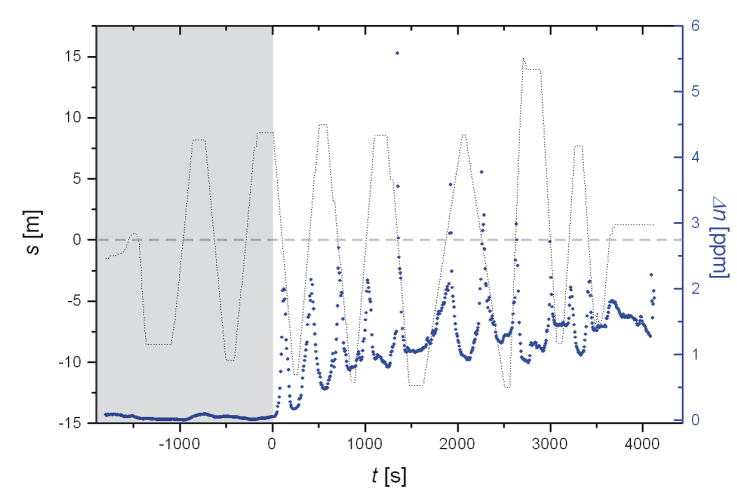

Figure 3: Chemical concentration measurements taken in a corridor in the university building with a robot moving at a constant speed. The gas sensor was placed at the front bumber approximately $30 \mathrm{~cm}$ in front of the robot's center of mass. A jar with an opening area of $20 \mathrm{~cm}^{2}$ filled with acetone was placed in the center of the corridor $(s=0 m)$ at the time $t=0 \mathrm{~s}$. The measured values are indicated by strong dots while the sensors position is drawn in with a thin dotted line.

front end. Up to now, this feature, which enables the halted robot to adjust the sensors, didn't produce any improvement because it turned out that the received signal gets considerably worse when the robot doesn't move. Therefore, the 'antennae' were used as a stiff expansion of the robot or, in other words, the possible sensor positions are approximately $-30 \mathrm{~cm},+30 \mathrm{~cm}$ and $+90 \mathrm{~cm}$ before the the robot's center of mass. Because only 3 MOX sensors were available the experiments were undertaken either using two sensors placed on the antennae or all three at one side.

\section{Results}

Some results are shown in figures 3 - 6. In each diagram the robot position is plotted against the time since the jar containing the liquid chemical was opened. The origin of the distance axis, indicated by a thick dashed line, denounces the position of the odour source. Sensor data, which were normalized to their base level, are plotted into the same graph against a second ordinate. Its scale was calibrated by measurements using a headspace sampler. Though it was not possible to reproduce exactly the same conditions in the laboratory as in the real world scenery, the stated values can only serve as a rough representation of concentration differences rather than abso- 


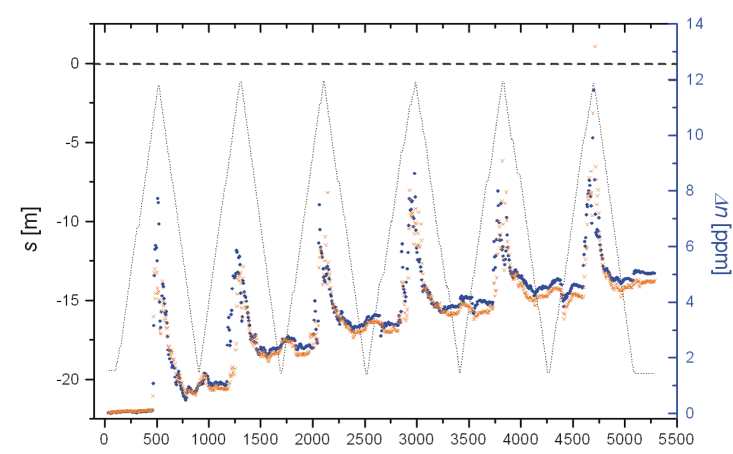

$t$ [s]

Figure 4: Chemical concentration measurements taken in a vacant appartement with a robot moving at a constant speed. The gas sensors were placed at the end of the robot's 'antennae' approximately $90 \mathrm{~cm}$ in front of the robot's center of mass. A jar with an opening area of $130 \mathrm{~cm}^{2}$ filled with ethanol was placed at the end of the corridor $(s=0 m)$ at the time $t=0 \mathrm{~s}$. The measured values are indicated by strong dots (right sensor) and crosses (left sensor) while the sensors position is drawn in with a thin dotted line.

lute values. Figure 3 shows the result of an experiment accomplished in the university corridor. In the lightly shaded left part of this diagram two runs up and down the corridor are shown which were recorded before the jar has been opened, whereas the right part shows several runs in the presence of the odour source placed at $s=0 \mathrm{~m}$. In all runs the robot moved with a constant speed $(5 \mathrm{~cm} / \mathrm{s}-20 \mathrm{~cm} / \mathrm{s})$. It can be seen that the robot clearly senses the presence of the analyte. In this case the smallest jar filled with acetone was used, while similar results were obtained using ethanol or one of the bigger jars. Notice that this result was achieved although the odour source was comparably weak and several people passed by in the course of this experiment.

Results of an experiment undertaken in the vacant appartement are shown in fig.4. The robot moved with a constant speed of $5 \mathrm{~cm} / \mathrm{s}$ and the jar $\left(130 \mathrm{~cm}^{2}\right)$ was placed at the end of the corridor. This figure shows the minor influence of the environment and that similar results were obtained using ethanol instead of acetone. Although the run of the curve is somewhat smoother, the general trend is similar to that shown in fig.3. Approaching the odour source leads to rising sensor values and the resulting peak clearly indicates the position of the maximum concentration of the analyte. Notice that the slow propagation of the volatile analyte is indicated by the ris-

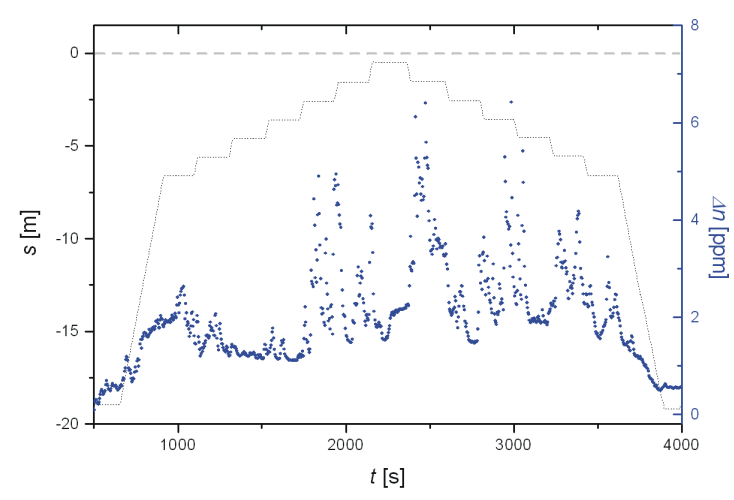

Figure 5: Chemical concentration measurements taken in a corridor inside a vacant appartement with a robot applying a stop-and-go strategy. The gas sensor was placed at the end of one of the robot's 'antennae' approximately $90 \mathrm{~cm}$ in front of the robot's center of mass. A jar with an opening area of $130 \mathrm{~cm}^{2}$ filled with ethanol was placed at the end of the corridor $(s=0 m)$ at the time $t=0 s$. The measured values are indicated by strong dots while the sensors position is drawn in with a thin dotted line.

ing baseline either in fig. 3 and in fig. 4 .

The robot moved at a constant speed during the just described investigations. In contrast to that, results of another run in which the robot executed a stop-and-go strategy can be seen in fig.5. The corresponding experiments were performed in the above mentioned vacant appartement using the biggest jar $\left(130 \mathrm{~cm}^{2}\right)$ filled with ethanol. It is obvious that this strategy is less suitable to solve the detection or even the localization problem. This might be caused by the working principle of metal oxide sensors which involves the consumption of some analyte material. If there is no air movement relative to the sensor the consumed analyte is replaced by molecular diffusion only. It is known that the diffusion velocities of gases are generally very slow [6]. Thus the saturation level of metal oxide gas sensors is significantly reduced if the transport of molecules is governed by diffusion. However, in a real world situation some weak airflow always exists caused by draft or convection flow. If the robot stops these rather arbitrary air movements dominate the received signal, superimposing that signals portion which contains the information on the analytes concentration.

Regarding fig.6 this effect appears even more striking. The diagram shows some subsequent runs which were carried out within the same experiment as shown in fig.5. If the robot moves forth and back 


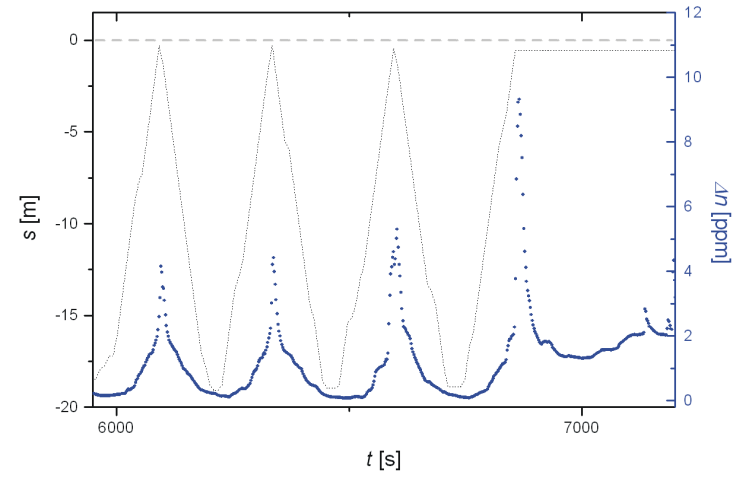

Figure 6: Chemical concentration measurements taken at the same experiment described in fig. 5 with a robot moving at a constant speed.

with a constant velocity of $15 \mathrm{~cm} / \mathrm{s}$ the run of the resulting curve is rather smooth indicating the location of the odour source by an outstanding peak. The last approach leads to a different behaviour: Immediately after the robot stops in front of the jar the sensor signal drops and the curve reveals acyclic oscillations.

\section{Localization Strategy}

If the robot drives over an odour source the resulting curve shows a distinct peak which indicates the point of maximum approximation between the gas sensor and the used jar (fig.3). It is therefore expected that a similar peak will arise if the robot is passing by within a certain distance. This distance is expected to be in the order of several meters because the full width at half maximum of the sustained peaks exceeded 5 meters even when the low intense odour source was used. Thus the following localization strategy seems to be reasonable: First the robot tries to examine the complete area in search of a peaked sensor signal. Considering the fact that driving with a constant speed is necessary to achieve meaningful sensor values, the examination of the environment should be preferably done by driving on straight lines. It is intended to use an algorithm developed by Kasper et al. [13] to perform the described area-wide search. Once the robot has recorded a discernible peaked curve, it fits the run of the curve by an appropriate function and returns to the center position of the identified peak. After being rotated by 90 degrees the robot examines the whole perpendicular line. If another peaked curve is detected, the middle of this peak is supposed to be the location of the odour source.

\section{Conclusion}

The presented results demonstrate that the described odour sensing system is able to detect volatile substances. The investigations showed that even weak odour sources can be sensed and that the influence of the environment is rather negligible.

The experiments revealed that the performance of the mobile odour sensing system could be significantly enhanced by driving the robot with a constant not too low speed, thus adding an extra airflow relative to the metal oxide sensors.

Furthermore a localization strategy was proposed which takes into account the special behaviour of the used gas sensors. It is intended to test the above drafted localization strategy in a wide rectangular environment like a gymnasium.

Within the scope of our investigations the sensors were tested at different positions. Although the results don't suggest a clearly favorable position a more exposed one seems to be preferable. Additional studies are needed to test other possible methods to improve the quality of the received sensor signal. First trials using different common pc fans yielded no considerable improvements but further experiments are needed to make concluding statements.

\section{References}

[1] A. Legin, A. Rudinitskaya, Y. Vlasov, C. Di Natale, F. Davide, and A. D'amico. Tasting of Beverages Using an Electronic Tongue Based on Potentiometer Sensor Array. Technical Digest of Eurosensors X, Leuven, Belgium, pages 427-430, 1996.

[2] J. W. Gardner. Pattern Recognition in the Warwick Electronic Nose. In 8th Int. Congress of the European Chemoreception Research Organisation, pages 9-, 1988.

[3] K. Persaud and G. Dodd. Analysis of Discrimination Mechanisms of the Mammalian Olfactory System Using a Model Nose. Nature, 299:352-355, 1982.

[4] A. Ikegami and M. Kaneyasu. Olfactory Detection Using Integrated Sensors. In Proceedings of the 3rd International Conference on 
Solid-State Sensors and Actuators (Transducers 85), IEEE Press, New York, pages 136139, 1985.

[5] J. Mitrovics, H. Ulmer, U. Weimar, and W. Göpel. Modular Sensor Systems for Gas Sensing and Odour Monitoring: The MOSES Concept. In ACS Symposium Series: Chemical Sensors and Interfacial Design, volume 31, pages 307-315, 1998.

[6] Takamichi Nakamoto, Hiroshi Ishida, and Toyosaka Moriizumi. A Sensing System for Odor Plumes. Analytical Chem. News \& Features, 1:531-537, August 1999.

[7] R. Andrew Russell, David Thiel, Reimundo Deveza, and Alan Mackay-Sim. A Robotic System to Locate Hazardous Chemical Leaks. In IEEE Int Conf. Robotics and Automation, pages 556-561, 1995.

[8] Titus Sharpe and Barbara Webb. Simulated and Situated Models of Chemical Trail Following in Ants. In R. Pfeifer, B. Blumberg, J.-A. Meyer, and S.W. Wilson, editors, Proceedings of the 5th Conference on Simulation of Adaptive Behaviour, pages 195-204, 1998.

[9] J. F. Engelberger. Robotics in Service. Kogan Page, London, 1989.

[10] R. Deveza, R. A. Russell, and D. Thiel. Robot Navigation by Smell: Problems and Solutions. In Proceedings of the Robots for Competitive Industries Conference, volume July, pages 458-466, Brisbane, 1993.

[11] Deveza, D. Thiel, R. A. Russell, and A. Mackay-Sim. Odor Sensing for Robot Guidance. The International Journal of Robotics Research, 3(13):232-239, June 1994.

[12] D. Osorio, W. M. Getz, and J. Rybak. Insect Vision and Olfaction: Different Neural Architectures for Different Kinds of Sensory Signal? In Proceedings of the 3rd Conference on Simulation of Adaptive Behaviour, pages 73-81, 1994.

[13] Michael Kasper, Dirk Müller, and Ewald von Puttkammer. Ein verhaltensorientierter Ansatz zum flächendeckenden Fahren in a priori unbekannter Umgebung. In Informatik aktuell, editor, Autonome Mobile Systeme (AMS), 14. Fachgespräch, pages 180-187, Karlsruhe, Germany, 30. November - 1. Dezember 1998. 